\title{
Seismic Analysis of Regular and Vertical Irregular RC Buildings
}

\author{
Sarfaraj Munshi and M.S. Bhandiwad
}

\begin{abstract}
Reinforced concrete multi storey buildings are subjected to severe earthquakes. It was found that the main reason for failure of $R C$ building is irregularity in its dimension and its lateral force resisting system. In this paper an analytical study is made to find response of different regular and vertical irregular structures such as mass, stiffness and geometry irregularity located in moderate zone III. Analysis has been made by taking 10 and 20 storey building by static and dynamic methods using ETABS 2013 and IS code 1893-2002 (part1). Behavior of structures will be found by comparing responses in the form of storey displacement, story drift and base shear for regular and vertical irregular structures. Different types of analysis methods such as equivalent static method, response spectrum and time history method are adopted. One is of regular structure and remaining are vertical irregular structural models. This paper shows that behavior vertical irregular structures as compared to regular structure.
\end{abstract}

Keywords--- Rc Building, Regular Building, Vertical Irregular Building, Equivalent Static Method, Response Spectrum Method, ETABS, IS 1893-2002 etc

\section{INTRODUCTION}

$\mathrm{E}^{\mathrm{A}}$ ARTHQUAKES are the most volatile, disturbing and unpredictable of all natural disasters, in which it is very difficult to save life and engineering properties. To overcome these problems we need to identify the seismic performance of various buildings through various analytical procedures. This is because to make sure that the various buildings withstand during earthquake events. And hence can save as many lives as possible. During earthquake the performance of a structure depends on many factors such as stiffness, adequate lateral strength, simple and regular configurations etc. The structures with regular geometry suffer less than the structures with irregular in their mass, stiffness, setback structures.

\section{A. Types of Irregularities \\ i. $\quad$ Plan Irregularities}

The irregularities that occur in their plan configuration of the building is termed as plan irregularities. This may due to re-entrant corners; diaphragm discontinuity, out-of-plane offsets etc.

Sarfaraj Munshi, M.Tech Student, Dept. of Civil Engineering, SDMCET, Dharwad, India. E-mail:sarfaraj3008@gmail.com

M.S. Bhandiwad, Assistant Professor, Dept. of Civil Engineering, SDMCET, Dharwad, India. E-mail:mallucv014@ gmail.com DOI:10.9756/BIJMMI.8178

\section{ii. $\quad$ Vertical Irregularities}

The structures with irregular distributions in their stiffness, mass and strength along the height of building is termed as vertical irregular. This may due to mass, stiffness, geometry, Discontinuity in Capacity, In-Plane Discontinuity etc.
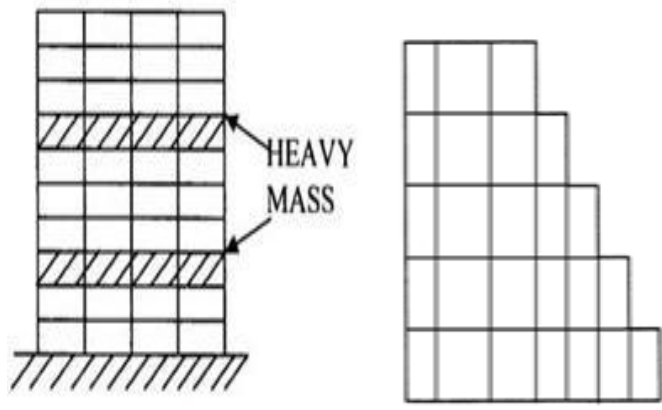

Mass Irregularities Geometry Irregularities
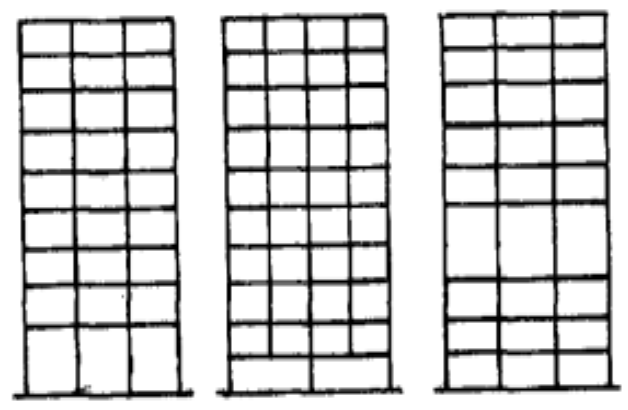

Stiffness Irregularities

Figure 1: Types of Vertical Irregularities

\section{B. Concept of Regular and Irregular Configuration}

To execute structure well in an earth quake it should have four main properties such as simple and regular and lateral strength, stiffness and ductility. IS Codes defined structural configuration either regular or irregular in terms of shape and size, structural and non-structural elements, and allocation of mass in the structures etc. A structure is said to be irregular if it possess at least one of the conditions in IS 1893:2002 part 1.

\section{Plan Irregularity}

Plan irregular or asymmetric buildings are those in which earthquake response is both translation as well torsional and it is as a result of stiffness and/or mass eccentricity of the building. Asymmetry in the structures exists in a normal symmetric building due to the indecision in the assessment of centre of mass, stiffness and imprecision in the measurement of the structural element dimensions. 


\section{Vertical Irregularity}

Vertical irregularity is due to the presence of uneven distribution of strength, stiffness and mass along the height of a building structure. Stiffness and mass irregularity are as a result of abrupt change in stiffness and mass between next floors lying above or below it respectively. Setback irregulity is as a result of abrupt change in lateral dimension of building.

\section{Methodology}

\section{A. Analysis Methods}

The word ETABS stands for extended 3D Analysis of Building Systems. This is Integrated Building Design Software. ETABS programs account the unique properties inbuilt in a arithmetic model of a structure which gives a computer demonstration to be constructed in the same way as that of real building. The terms used in this are beam, column, brace, and wall, other than FE and nodes. ETABS gives computerization and particular options for the process of model formation, analysis, and design fast, easy and convenient. It provides tools for drawing floor framing, frames, columns and walls either steel or concrete.

\section{i. $\quad$ Time History Analysis}

Time history evaluation methods contain the stepwise answer within the time domain of the multi degree-of-freedom equations of movement which symbolize the genuine reaction of a structure. It's the most advanced evaluation procedure to be accessible to a structural engineer. Its answer is an instantaneous function of the earthquake floor motion chosen as an enter parameter for a particular building.

\section{ii. Response Spectrum Analysis}

This technique enables the more than one modes of response of a construction to be taken into consideration and this is necessary in lots of building codes for all but excluding very simple or very complex buildings. Following diagram shows the standard response spectrum for medium soil type and that can be given in the form of time period versus spectral acceleration coefficient $(\mathrm{Sa} / \mathrm{g})$.

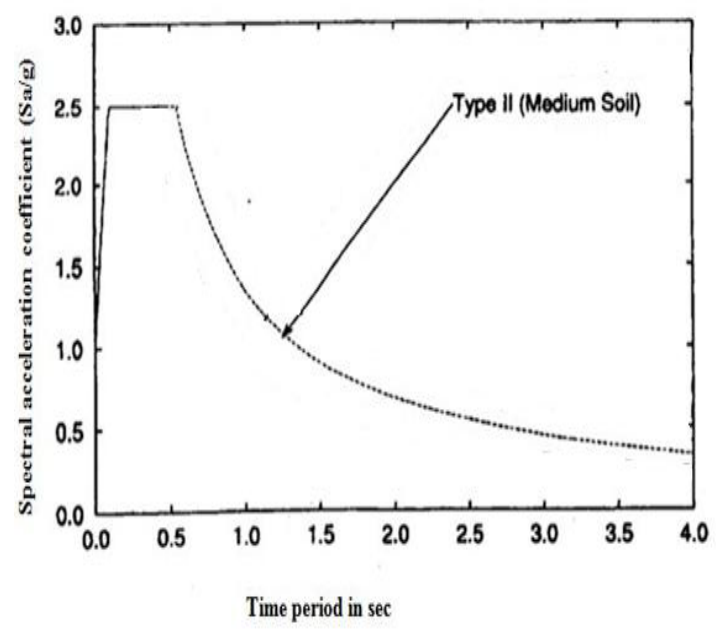

Figure 2: Response Spectrum for Medium Soil Type for 5\% Damping

\section{iii. Equivalent Static Analysis}

It is elastic design method. In any case it is easy to use than the multi-model response approach, with the utmost simplifying assumptions being questionably more regular with other assumptions absolute in other places within the design process.

\section{A. Building Modeling}

\section{i. Building Description}

The modeling of the building is done using ETABS version 13.1 software. For my work a 3 dimensional 10 and 20 storeyed building considered with 3 bays along $\mathrm{X}$ direction and 6 bays along $\mathrm{Y}$ direction for regular, vertical mass irregular and vertical stiffness irregular structures. For vertical geometry irregular 8 bays along $\mathrm{X}$ direction and 6 bays along $\mathrm{Y}$ direction is considered.

Table 1: Building Description

\begin{tabular}{|l|l|}
\hline Particulars & Reinforced Concrete Building \\
\hline Plan dimension & $15 \mathrm{mX} 24 \mathrm{~m}$ \\
\hline Height of buildings considered & $35 \mathrm{~m}, 70 \mathrm{~m}$ \\
\hline Grade of steel & $\mathrm{Fe} 415$ \\
\hline Grade of concrete & $\mathrm{M} 25$ \\
\hline No. of storey & 10,20 \\
\hline Beam size & $300 \mathrm{mmX} 600 \mathrm{~mm}$ \\
\hline Column size & $500 \mathrm{mmX} 500 \mathrm{~mm}$ \\
\hline Soil type & Medium(II) \\
\hline Seismic zone & III \\
\hline
\end{tabular}

\section{ii. $\quad$ Plans and Models}

Plans and 3D models considered for the analysis of regular and irregular buildings. The following are the plans for regular and irregular building.

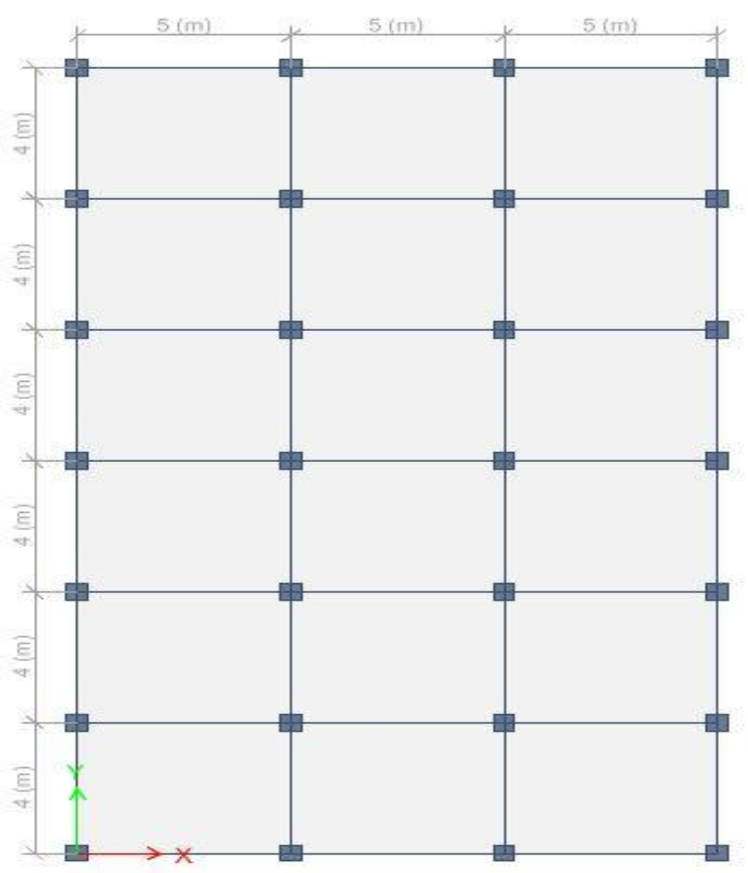

Figure 3: Plan of Regular, Mass Irregular and Stiffness Irregular Building 


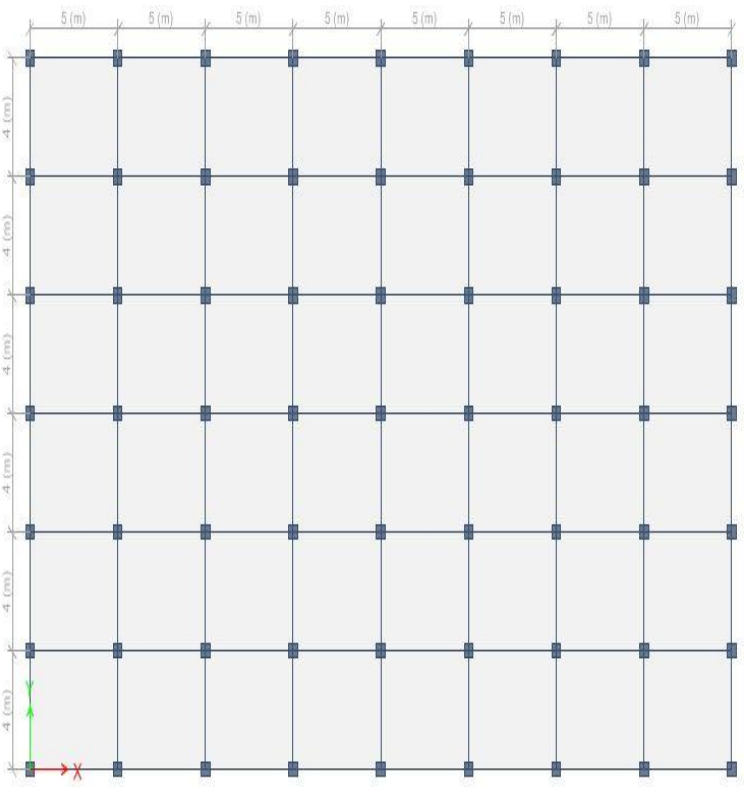

Figure 4: Plan of Geometric Irregular Building

III. RESULTS AND DISCUSSIONS

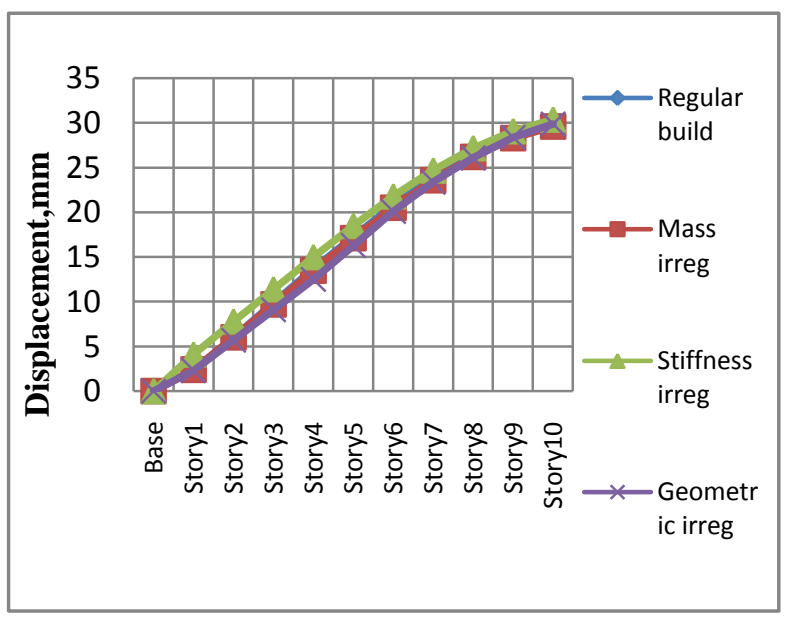

Figure 5: Comparison of Displacement in X Direction for All Types of Buildings by ESA

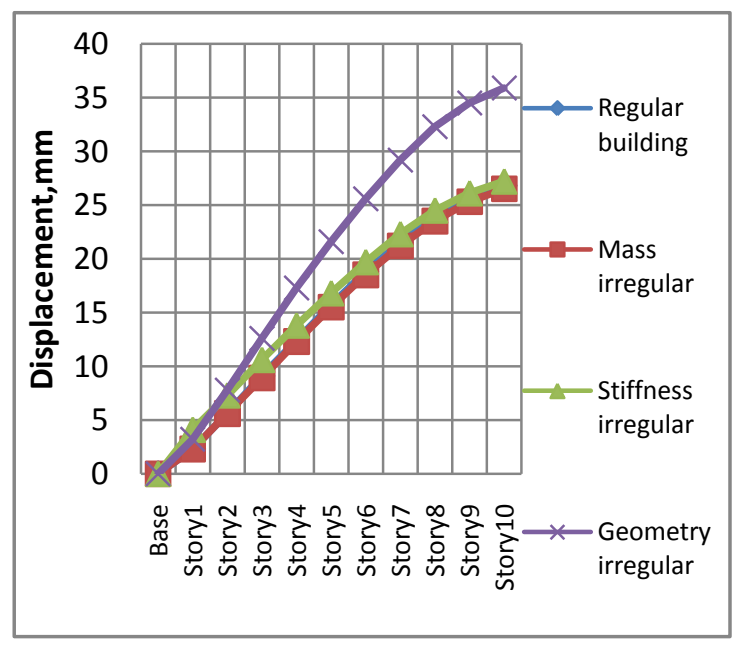

Figure 6: Comparison of Displacement in Y Direction for All Types of Buildings by ESA

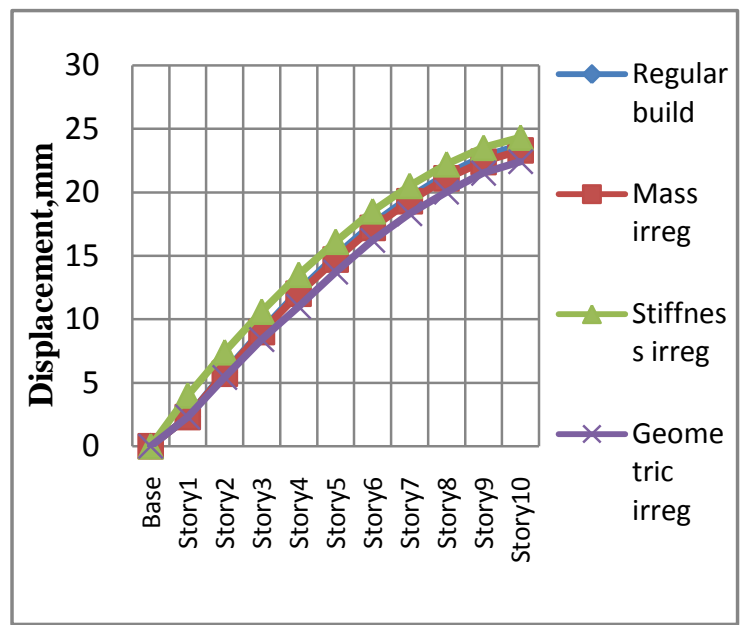

Figure 7: Comparison of Displacement in X Direction for All types of Building by RSA

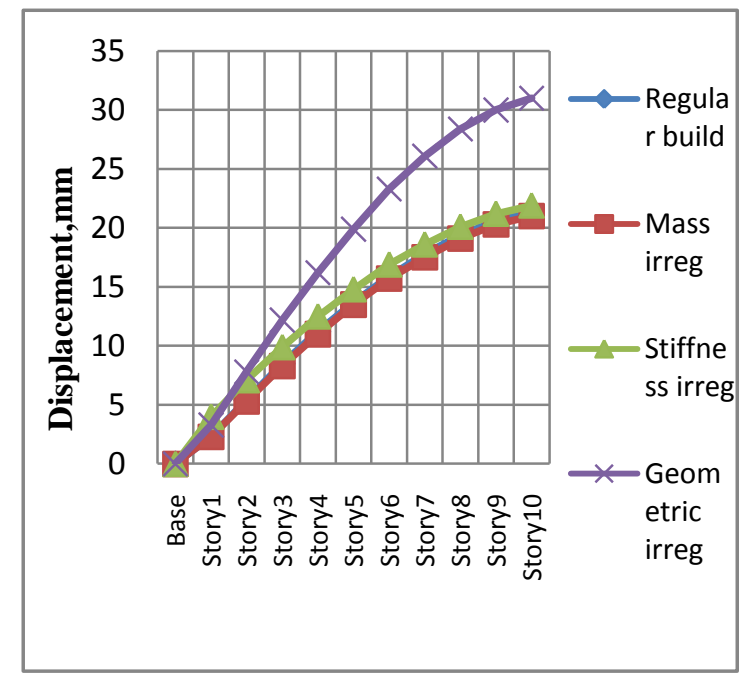

Figure 8: Comparison of Displacement in Y Direct for all Types of Buildings by RSA

\section{CONCLUSION}

Three types of irregularities namely mass irregularity, stiffness irregularity and vertical geometry irregularity were considered. Equivalent static analysis (ESA), Response spectrum analysis (RSA) was conducted for each type of irregular buildings and the storey displacement and drift obtained and were compared with that of a regular structure.

1) It is observed that the stiffness irregularity structures show $41.4 \%$ increase in the displacement (fig. 5) of stiffness building at first floor along $\mathrm{x}$ direction and that of setback buildings show $25.35 \%$ along $y$ direction (fig. 6) compared to regular building.

2) According to the results of Response spectrum analysis, the displacement along $\mathrm{x}$ direction is more in case of stiffness irregular building as compared to regular building. There is $46.4 \%$ increase in displacement (fig.7). 
3) By RSA method along y direction the geometry irregularity structures show more displacement i.e. at $7^{\text {th }}$ storey $31.63 \%$ increase in displacement than regular structures as shown in fig 8 .

4) Therefore the performance of irregular structure is low compared to that of the regular structure in earthquake prone areas.

\section{REFERENCES}

[1] A.G. Costa, C.S. Oliviera and R.T. Duartze, "Influence of Vertical Irregularities on Response of Buildings", 1998.

[2] M. Shahrooz Bahrain and P. Moehle Jack, "Seismic Response and Design of Setback Buildings-Journal of Structural Engineering", Vol. 116, No. 5, Pp. 1423-1439, 1990.

[3] P. Sarkar, A.M. Prasad and D. Menon, "Vertical geometric irregularity in stepped building frames. Engineering Structures, Vol. 32, No. 8, Pp. 2175-2182, 2010.

[4] E. V. Valmundsson and J.M. Nau, "Seismic response of building frames with vertical structural irregularities", Journal of Structural Engineering, Vol. 123, No. 1, Pp. 30-41, 1997.

[5] T.L. Karavasilis, N. Bazeos and D.E. Beskos, "Estimation of seismic inelastic deformation demands in plane steel MRF with vertical mass irregularities. Engineering structures, Vol. 30, No. 11, Pp. 3265-3275, 2008.

[6] IS 456-2000-Design of RC structures.

[7] IS: 1893-2002 (Part 1) Criteria for Earthquake Resistant Design of Structures, part 1-General provisions and buildings, fifth revision, Bureau of Indian Standard, New Delhi, India. 